\title{
Priority Based Energy Aware (PEA) Routing Protocol for WBANs
}

\author{
Sadaf Talha, Rizwan Ahmad and Adnan Khalid Kiani \\ School of Electrical Engineering and Computer Science (SEECS) \\ National University of Sciences and Technology (NUST) \\ H-12 Campus, Islamabad, Pakistan. \\ \{12mseestalha, rizwan.ahmad, adnan.khalid@ seecs.edu.pk\}
}

\begin{abstract}
Wireless Body Area Networks (WBANs) is a new technology for remote monitoring of patients. Sensor nodes are placed on different parts of the body such as implants and on body to collect data and transfer to the Sink node. Change in body posture, placement of sensors, priority of sensor data and energy consumption makes routing very difficult. Therefore, a Priority based Energy Aware (PEA) routing protocol is proposed in this paper. Child nodes choose a parent node connected to Sink based on a cost function that depends upon priority, residual energy and distance of node. Residual energy facilitates load balancing i.e. selection of different nodes for transmission. Distance helps in successful packet delivery to the parent node and caters for body postures. Priority helps to select a best possible path to forward the critical data keeping in view the energy constraint in WBANs. Comparison of different cost functions with proposed PEA protocol for performance metrics such as network lifetime, throughput and residual energy reveals that the proposed protocol results in increased network lifetime, throughput improvement of around $50 \%$ and higher residual energy.
\end{abstract}

\section{INTRODUCTION}

Recent advances in sensor technology and wireless communications have led to the advent of a new technology named as Wireless Body Area Networks (WBANs). It has brought a major revolution in the health sector and monitoring of patients. Different low power, invasive, non-invasive nodes are placed either inside or on the body to detect different parameters that helps in remote monitoring of patients. A recently released standard specifies up to $10 \mathrm{Mbps}$ data rates that can be used to accurately fulfill the health services requirements [1] [2]. By placing the nodes in correct positions on human body, accurate information can be achieved. The nodes are basically the intelligent psychological sensors that collect information from different parts of body [3]. This provides a cheaper and an effective way to share regular updates with the physician or remotely monitor the patient.

A human body has a very complex structure consisting of tissues and organs due to which the signal/data packet might be lost or is received with a delay. In order to provide efficient observation and communication, nodes are placed either inside the human body or on human body depending upon the information required [4]. These nodes communicate with each other and eventually forward data to Sink node which is the central coordinator in WBANs. The role of the Sink node is that of a main processing unit that gathers the information from all the nodes placed on different parts of body. It is further responsible to transmit the integrated information to the external gateway of OFF body node and communication with other network infrastructure [4] [5].

An important challenge in WBANs is the movement human body. Human body frequently changes its posture and is not static for long time. Due to the complex internal structure, it might be possible that a certain posture results in blockage of signal from being transmitted to sink node [6]. Therefore, while devising a routing strategy it is important to cater for the posture change of body without affecting the overall communication process.

Another major concern in WBANs is the energy consumption. As the nodes are placed in/on human body therefore, batteries of nodes cannot be frequently recharged or replaced. The nodes have to both transmit and receive the data which might result in quick energy depletion of nodes if proper routing technique is not implemented [7].

The sensors placed on human body collect different information e.g. glucose, pulse monitoring, heart rate, foot pressure etc. Some of the information is very critical and needs to be monitored frequently. This is achieved through prioritizing the nodes [1]. For example, for a diabetic patient, monitoring of glucose at regular intervals is necessary to avoid any heart stroke [8]. Data of nodes with high priority is required to be forwarded reliably and without information loss. As this node forwards its data more frequently than other, it might result in energy depletion if proper communication strategy is not adopted. All these issues make routing a big challenge for a very small WBAN. The factors mentioned above, point to the requirement of a routing strategy that effectively communicates the data of all nodes to central coordinator thus catering for the body postures, that provides energy efficient solution to address the major energy concerns of WBANs and that also takes priority data into account.

In [9] author presents a routing strategy having different nodes with different energies. A cluster head $(\mathrm{CH})$ is selected as a node having maximum energy. There is equal probability of being selected as a $\mathrm{CH}$ in each round. A schedule is shared by $\mathrm{CH}$ to remaining nodes for transmitting their data. In this paper, priority of data is not addressed. High priority data 
node would have to wait for its turn to transmit the data. A cost function depending on received and transmitted power is presented in [10]. This approach assumes all nodes have equal priority which is not a realistic assumption. In [11] authors present a routing protocol in which a forwarder is used to transmit data to Sink. The forwarder is selected on the basis of cost function having minimum distance with sink and maximum residual energy. It collects data from all nodes and then forwards to Sink. Collecting data from all nodes result in collision and transmission delay to Sink. Nodes with high priority can send directly to Sink, however, priority is not used in cost function. All of the above routing protocols donot cater the affect of body posture change.

In this work, a routing scheme called Priority based Energy Aware (PEA) is proposed that effectively transfers the data to sink node. The nodes in the network are categorized as child nodes, parent nodes and sink. Child nodes forward their data to parent node directly or by using another child node. The selection of child node is made by computing a cost function based on energy, distance and priority of nodes data. This division of nodes addresses the issue of energy constraint. It is a two hop transmission mechanism that would transmit the signal using less energy. Postural changes are also catered by relay child nodes taking into account the distance between nodes and different parent nodes. Child nodes will only select the parent node that is nearest to it during transmission.

Section II describes the system model in detail and discusses the network topology. Section III describes the proposed protocol PEA and section IV provides a comparison of different cost functions with proposed routing protocol technique and network lifetime, residual energy and throughput comparisons. Finally section V provides conclusion.

\section{SYSTEM MODEL}

In this proposed scheme, routing based protocol is presented in WBANs to show how to effectively communicate between nodes placed on different parts of body. The scope of this work is based on communication within the body and the communication of sink with off body infrastructure is not considered here. The nodes placed in/on human body are divided into sink, parent and child nodes. Sink: It is the main central coordinator having maximum energy and lifetime. All the nodes transfer their data to this controlling node. Parent node $(P N)$ : It acts as a relay for child nodes to forward their data to the Sink. They have sufficient energy to forward the transmission to sink directly. Child node $(C N)$ : These nodes are minimum energy nodes that collect data from different parts of body and forward to parent nodes.Our topology contains a Sink node and 8 remaining nodes out of which 3 act as PNs while remaining 5 as $C N s$. Placement of the nodes is shown in fig. 1. Initially, all nodes broadcast a small HELLO packet indicating its position on body, priority and energy level. In this way, all $C N s$ would be able to know about the PNs which in turn will know about the Sink node location. The $P N-1$ controls the $C N s$ from $1-3, P N-2$ controls $C N s-4,5$ whereas $P N-3$ does not have any child. Our main focus is to design

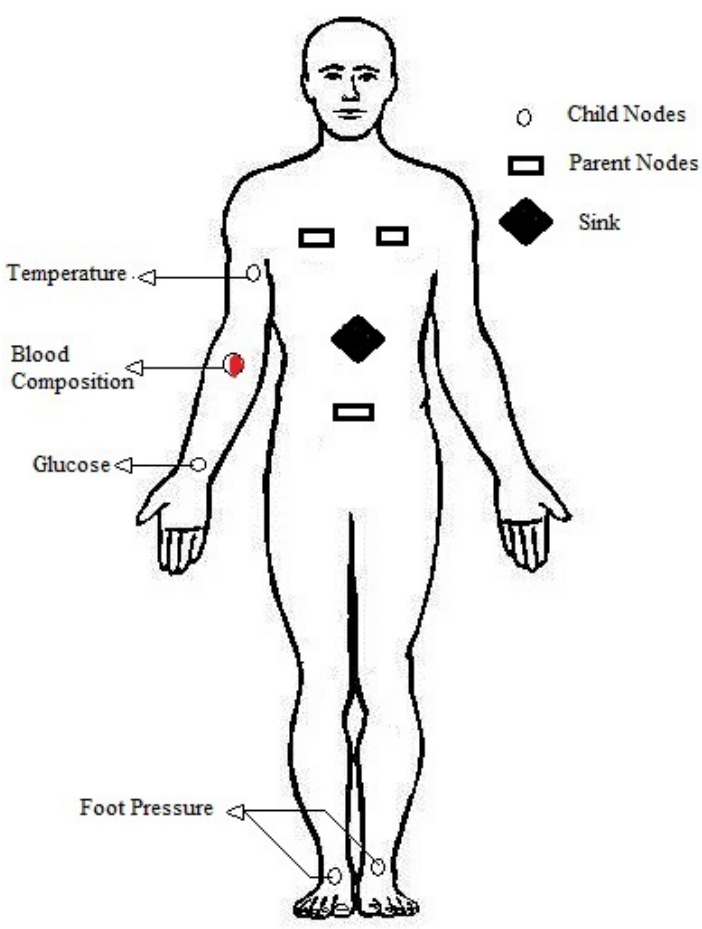

Fig. 1. Node Deployment on human body.

a communication strategy of $P N-1$ with its child nodes. It is possible that due to posture change child nodes of $P N 1$ can use $P N 2$ or $P N 3$ as parent nodes. All the child nodes have different priority based on the data they carry. $C N-1,4$ and 5 have lowest priority, whereas, $C N-3$ has the highest priority because it carries the most critical data. Priority of $\mathrm{CN}-2$ is in between the two. The priority mainly play role in selection of $C N-1$ and $C N-2$ as relay by $C N-3$. As $C N-1$ is near to $P N-1$ which means that, there are more chances of its selection as relay if priority is not considered resulting in depletion of $\mathrm{CN}$ 1 first and then using $C N-2$ as relay. This provides selection of diverse link.

$\mathrm{CN}-3$ is the node having highest priority as it carries the critical data. This node actually detects the posture change of body. Based on the posture at the time of forwarding, it selects its relay/forwarder node. As nodes know about the position of other $C N s$ and $P N s$, based on which they can calculate the distance from each other. Main factor in detecting the posture of body is the distance between $C N-3$ with relay, $P N-1$ and $P N-2$. Two different body postures are shown in fig. 2 where fig. 2a shows a posture with hands near $P N-1$, distance of $\mathrm{CN}-3$ with $P N-1$ is also provided. While in fig. $2 \mathrm{~b}$ hands are near $P N-2$ and the distance of $C N-3$ with $P N-2$ is shown. The inward or outward movement of right hand also changes the selection of relay/forwarder. $C N-4$ and $C N-5$ are used to note the foot pressure. They use $P N-2$ to forward the data to sink. $P N-3$ is placed near heart. It measures heart beat rate and forwards to sink. Energy of every node will be decreased 


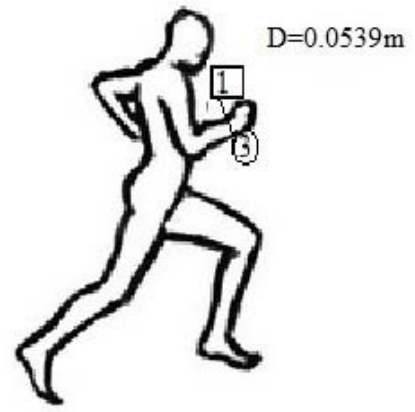

(a)

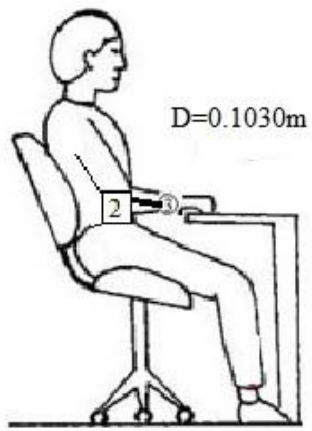

(b)
Fig. 2. Body Posture Change.

after every transmission and receiving of packet. The energy depletion of each node is given by equations (1) and (2).

$$
\begin{gathered}
E_{T x}=E_{T E} \times b+E_{A} \times b \times d^{2} \\
E_{R x}=E_{R E} \times b
\end{gathered}
$$

Where $E_{T x}$ and $E_{R x}$ is the energy during transmission and reception of packet, $E_{T E}$ and $E_{R E}$ shows the energy for electronic circuit of transmitter and receiver and $E_{A}$ is energy for amplifier circuit. Packet size is variable $b$ and $d$ is the distance. Values of these parameters depend on the hardware used.

\section{PROPOSED PROTOCOL: PEA}

In first phase, a relay would be selected out of and $\mathrm{CN}$ 2 which would be used to forward the data of both nodes to parent node. Equation 3 shows the cost function based on which relay is selected among $C N-1$ and 2 to forward the data to $P N-1$. The one with maximum cost function would be chosen to carry the data of both $C N s$ to $P N-1$. Here $E(i)$ represents the residual energy of ith node, $D(i)$ is the distance of ith node with the $P N-1$ and $P(i)$ shows the priority. $E(i)$ of node should be high in order to effectively forward the data. $D(i)$ between child node and parent node should be minimum so that the data packet is more likely to be sent to the receiver without suffering much path loss. The node with high $P(i)$ means that it carries more important data. If $\mathrm{CN}-3$ uses relay, it computes the cost function of both paths i.e. $\mathrm{CN}-3$ to relay and relay to $P N$.

$$
\text { CostFunction }(i)=\frac{E(i)}{D(i)}+P(i)
$$

In this paper, we compare performance of proposed cost function with following base cost functions shown in equations (4) and (5) and cost function from [11] shown in equation (6). Same network topology is considered for fair comparison of all the cost functions.

$$
\text { CostFunction }(i)=E(i)
$$

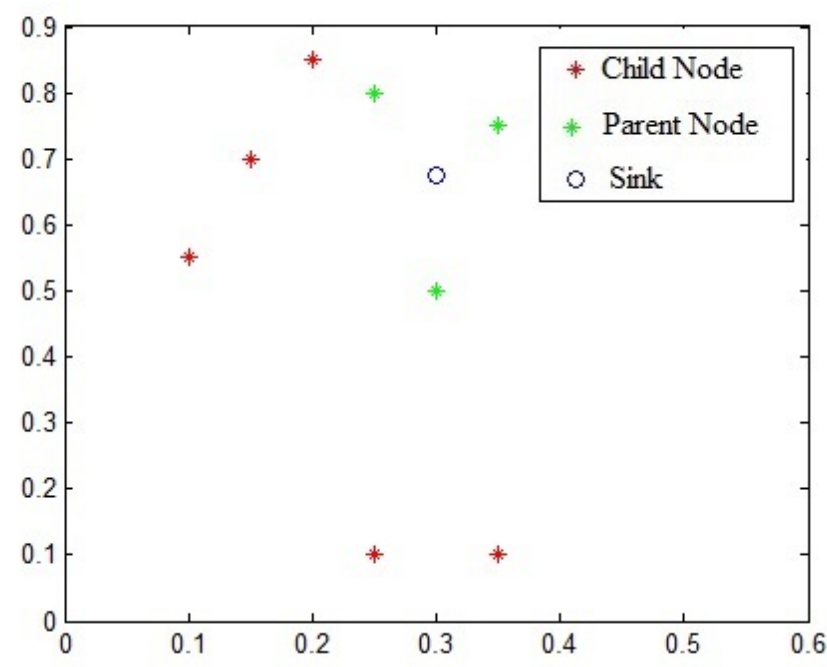

Fig. 3. Simulation Topology.

$$
\begin{aligned}
& \text { CostFunction }(i)=D(i) \\
& \text { CostFunction }(i)=\frac{D(i)}{E(i)}
\end{aligned}
$$

Maximum residual energy cost function in equation (4) suggests that relay should be selected on the basis of maximum residual energy only. The node with maximum $\mathrm{E}$ would forward the data to the $P N-1$. A node would be considered as dead when its energy falls below a certain threshold. In minimum distance cost function of equation (5), only distance $D(i)$ between two nodes is used to select the relay. Node with minimum distance with $P N-1$ would be chosen as a relay. As the position of $C N-1, C N-2$ and $P N-1$ is fixed, therefore, the distance will always remain fixed. This means that initially only $\mathrm{CN}-1$ would be selected as relay. $\mathrm{CN}-2$ will be used as relay only when $C N-1$ becomes a dead node after performing multiple transmissions. Cost function shown in equation (6) is based on SIMPLE protocol [11] which proposes to use minimum $D$ and maximum $E$ of nodes in the relay selection process. Node with minimum cost function would be selected as relay.

$\mathrm{CN}-3$ being the highest priority node carries the most critical data. Its movement is actually the deciding factor for body posture. During its transmission, it checks its distance from relay, $P N-1$ and $P N-2$. It forwards the data to the one with which its distance is minimum. Fig. 3 shows the simulation topology of proposed protocol while fig. 4 shows how different body postures affect the selection of relay. Dotted arrow indicates the new position of $C N-3$. Fig. 4a elaborates the scenario in which a relay among $C N-1$ and $C N-2$ is used to forward the data of $C N-3$ to $P N-1$. This might be a sleeping posture in which right hand is placed below the cheek. In fig. $4 \mathrm{~b}$, a body is in sitting position with its hands under the face. This node movement can also be considered as a running posture. In both scenarios, $\mathrm{CN}-3$ becomes nearer to $P N-1$ and 


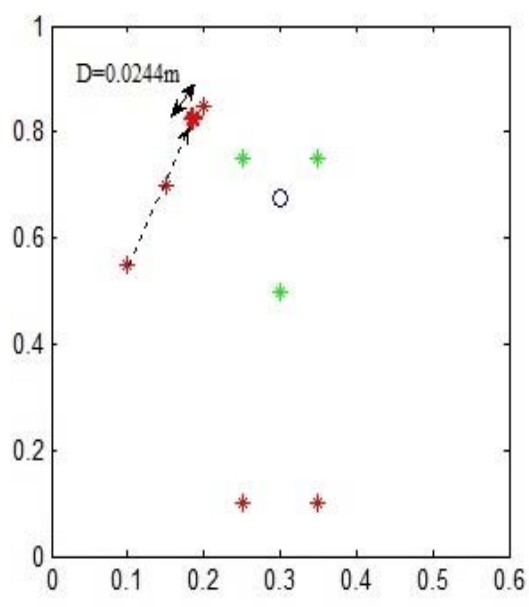

(a) Sleeping Posture

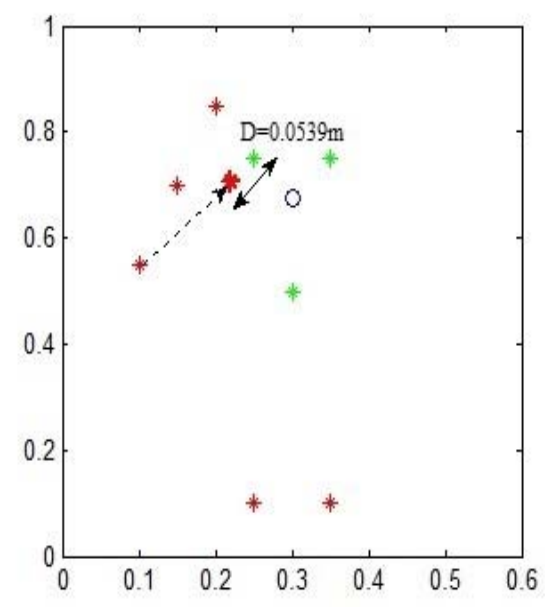

(b) Running Posture

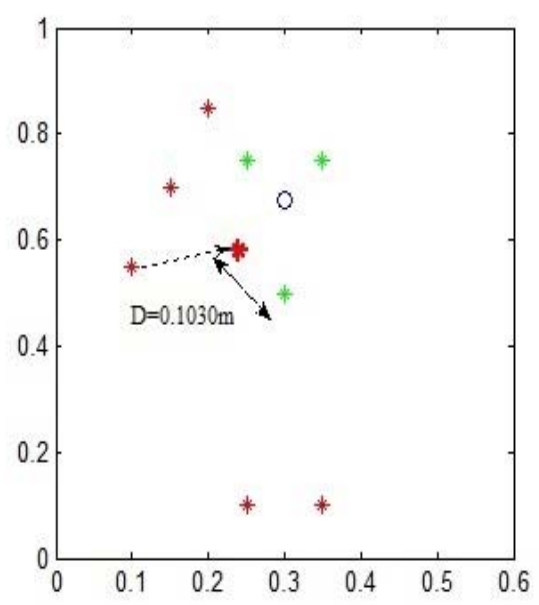

(c) Sitting Posture

Fig. 4. Body Postures Topologies.

TABLE I

SIMULATION PARAMETERS.

\begin{tabular}{|l|c|r|}
\hline Parameter & Value & Unit \\
\hline$D C$ Current $(T x)$ & 10.5 & $\mathrm{~mA}$ \\
\hline$D C$ Current $(R x)$ & 18 & $\mathrm{~mA}$ \\
\hline$E_{T E}$ & 16.7 & $\mathrm{~nJ} / \mathrm{bit}$ \\
\hline$E_{R E}$ & 36.1 & $\mathrm{~nJ} / \mathrm{bit}$ \\
\hline$E_{A}$ & 1.97 & $\mathrm{~nJ} / \mathrm{bit}$ \\
\hline$E$ & 0.5 & $\mathrm{~J}$ \\
\hline
\end{tabular}

selects it as a forwarder. Fig. 4c represents the sitting posture with hands on table. In this case, $P N-2$ is selected as relay as the distance is least.

\section{PERFORMANCE ANALYSIS}

For simulation of results, MATLAB 2013a is used. Parameters used for simulation are shown in Table.1. Network topologies shown in fig. 3 and 4 are used for the simulation and body posture changes. Performance metrics inspired from [11] for evaluation of proposed routing scheme are explained below. Simulation is run for 8000 rounds. The impact of different metrics on cost functions explained in Section III are shown.

\section{A. Dead Nodes}

When energy of node becomes less than the threshold, it is declared as a dead node. Fig. 5 depicts the performance comparison in terms of network lifetime for all four cost functions. Here, PEA routing performs better than rest of the cost functions. All five nodes die out quickly for the maximum residual energy cost function. This result in small network lifetime as the node having maximum residual energy are selected as relays making the nodes to die out before other cases. By using minimum distance cost function as a parameter for selection of relay, first node start to die before others because initially only $C N-1$ would be selected as a relay to carry the

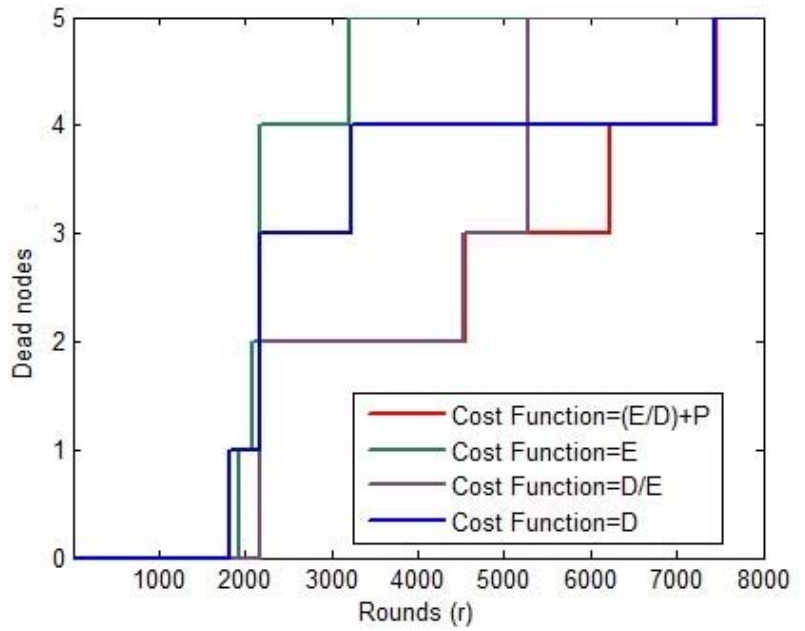

Fig. 5. Network Lifetime.

data. When its energy depletes completely, only then $\mathrm{CN}-2$ would take the role of relay. In SIMPLE cost function, where energy and distance both are used, performance is same as PEA till 3 nodes but remaining 2 nodes die quickly making the network lifetime short. Maximum energy cost function barely survives till 3200 rounds, followed by minimum distance. SIMPLE survives till 5200 rounds and both minimum distance and PEA reaches 7500 rounds with equal initial energy of all nodes.

\section{B. Throughput}

Throughput is defined as the total successful packets received at sink till the energy of node is completely depleted. Fig.6 shows the comparison of all four cost functions in terms of throughput. With SIMPLE cost function, initially 


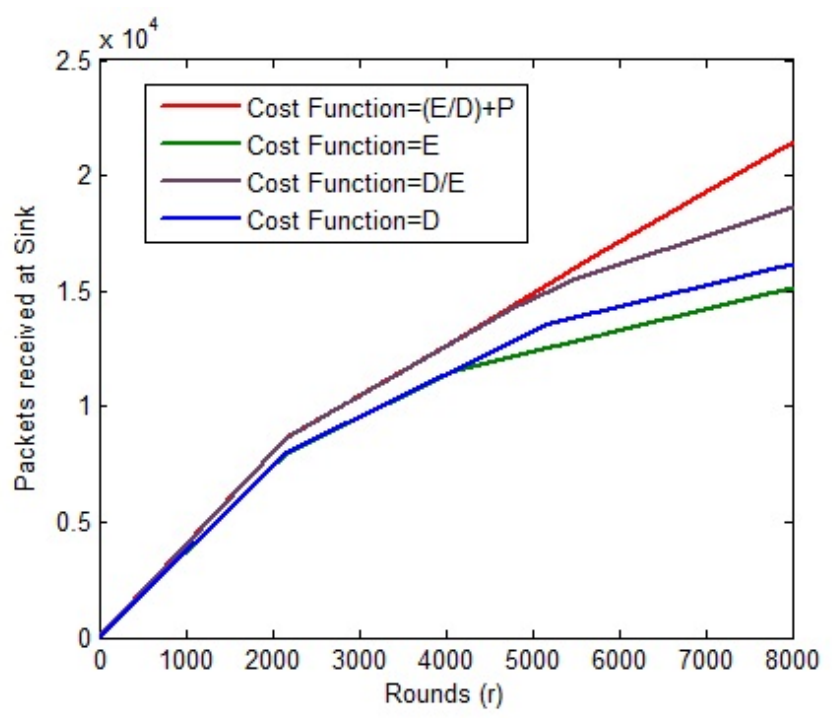

Fig. 6. Throughput.

significant numbers of packets are received but they start to reduce at around 5000 rounds because the nodes start to die out. Similarly for maximum energy cost function throughput is the worst among all because the node with maximum residual energy will forward all the transmissions resulting in energy depletion of that node. PEA completely outperforms all other schemes under consideration with highest throughput. Result at 6000 rounds show that PEA provides throughput improvement of $18 \%, 12 \%$ and $6.25 \%$ over maximum energy, minimum distance and SIMPLE respectively. Overall PEA can result in throughput improvement of around 50\% at 8000 rounds over the cost function considering maximum energy.

\section{Residual Energy}

Residual energy indicates the average energy comsumed in each round. Fig. 7 shows the residual energy of nodes for all cost functions. The worst results are obtained by using the maximum energy cost function because the nodes with maximum residual energy would be transmitting the data frequently resulting in quick energy depletion. PEA again outperforms others after 3500 rounds. At 5000 rounds, PEA shows improvement of almost $20 \%$ over maximum energy cost function.

\section{CONCLUSION}

In this paper, PEA routing protocol is proposed which attempts to address the major routing issues of WBANs. It uses a cost function to select a relay to forward the data to sink node. This cost function includes residual energy, distance and priority of data node. In order to cater the effect of posture change, value of distance is used to forward the data to the best parent node at the time of transmission. Performance evaluation based on network lifetime, throughput, and residual energy show that PEA clearly outperforms the other techniques. In future, we plan to consider more complex

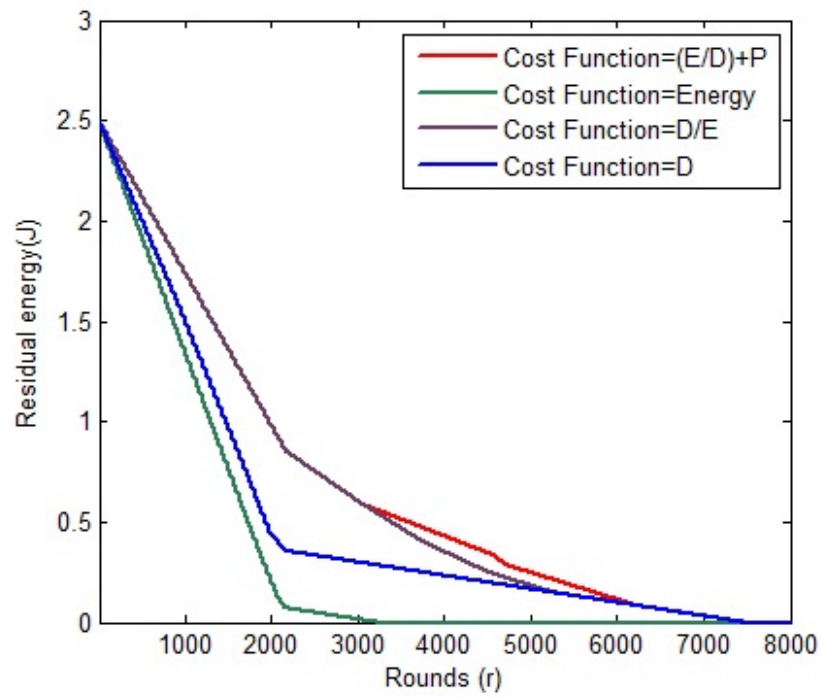

Fig. 7. Residual Energy Analysis.

scenarios, work on parent nodes with different priorities and the possibility of collision between $P N$ and Sink.

\section{REFERENCES}

[1] IEEE Standard for Local and metropolitan area networks, Part 15.6: Wireless Body Area Networks, IEEE Std 802.15.-2012.

[2] E. Jovanov, A. Milenkovic, C. Otto and P. C. D. G, A wireless body area network of intelligent motion sensors for computer assisted physical rehabilitation, Journal of Neuro Engineering and Rehabilitation 2005.

[3] M. Quwaider, J. Rao, and S. Biswas,, Body-posture-based dynamic link power control in wearable sensor networks, IEEE, Communications Magazine, 2010.

[4] E. Rebeiz, G. Caire, A.F. Molisch, Energy Delay Tradeoff and Dynamic Sleep Switching for Bluetooth Like Body Area Sensor Networks, IEEE Transactions on Communications, VOL. 60, NO. 9, September 2012.

[5] H. Chebbo, S. Abedi, T. A. Lamahewa, D. B. Smith, D. Miniutti, L. Hanlen, Reliable Body Area Networks Using Relays: Restricted Tree Topology, IEEE Workshop on Computing, Networking and Communications, 2012.

[6] I. C. Paschalidis, W. Dai, D.Guo, Posture Detection with Body Area Networks, Proceedings of the 6th International Conference on Body Area Networks, pg. 27-33, 2011.

[7] S. Hayat, N. Javaid, Z. A. Khan, A. Shareef, A. Mahmood, S. H. Bouk, Energy Efficient MAC Protocols, IEEE 14th International Conference on High Performance Computing and Communications, 2012.

[8] S. Ullah, M. Imran, and M. Alnuem, A Hybrid and Secure PriorityGuaranteed MAC Protocol for Wireless Body Area Network, International Journal of Distributed Sensor Networks Volume 2014 (2014), Article ID 481761.

[9] G.R. Tsouri, A. Prieto, N. Argade, "On increasing network lifetime in body area networks using global routing with energy consumption balancing", Sensors, 12 (10) (2012), pp. 1308813108.

[10] W. R. Heinzelman, A. Chandrakasan, and H. Balakrishnan, EnergyEfficient Communication Protocol for Wireless Microsensor Networks, Proceedings of the 33rd Hawaii International Conference on System Sciences, IEEE, 2000.

[11] Q. Nadeem, N. Javaid, S. N. Mohammad, M. Y. Khan, S. Sarfraz, M. Gull, SIMPLE: Stable Increased-throughput Multi-hop Protocol for Link Efficiency in Wireless Body Area Networks, IEEE Broadband and Wireless Computing, Communication and Applications (BWCCA), 2013. 